\title{
HYPERSPECTRAL APPLICATIONS IN THE GLOBAL TRANSPORTATION INFRASTRUCTURE
}

\author{
Raj Bridgelall $^{a}, J_{\text {. Bruce Rafert }}^{b}$, Denver Tolliver $^{a}$ \\ ${ }^{a}$ North Dakota State University, Upper Great Plains Transportation Institute, Fargo, ND, U.S.A. \\ ${ }^{\mathrm{b}}$ Department of Physics, North Dakota State University, Fargo, ND, U.S.A.
}

\begin{abstract}
Hyperspectral remote sensing is an emerging field with potential applications in the observation, management, and maintenance of the global transportation infrastructure. This study introduces a general analytical framework to link transportation systems analysis and hyperspectral analysis. The authors introduce a range of applications that would benefit from the capabilities of hyperspectral remote sensing. They selected three critical but unrelated applications and identified both the spatial and spectral information of their key operational characteristics to demonstrate the hyperspectral utility. The specific scenario studies exemplifies the general approach of utilizing the outputs of hyperspectral analysis to improve models that practitioners currently use to analyze a variety of transportation problems including roadway congestion forecasting, railway condition monitoring, and pipeline risk management.
\end{abstract}

Index Terms - Hyperspectral image processing, intelligent transportation systems, remote sensing, smart infrastructure, unmanned aircraft systems

\section{INTRODUCTION}

While hyperspectral remote sensing has rapidly emerged as a mature field since the early 2000's [1], relative few applications of hyperspectral analysis in transportation currently exist. As of January 2015, there were only 14 applications reported in the worldwide literature [2]. Early research concluded that the high uncertainty and latency of results from existing airborne and spaceborne platforms create major impediments to the widespread use of remote sensing for applications in transportation [3]. Nevertheless, the growing popularity and capabilities of small, low-cost unmanned aircraft systems (UAS) coupled with the steady advancement of lightweight hyperspectral imager payloads of decreasing cost promises vast improvements in mobility, accessibility, spatial resolution, and image quality. However, the government regulations and privacy concerns of many countries continue to hinder the deployment of UAS for commercial applications [4]. Even at the conclusion of rulemaking and policy enactment, the global transportation industry will need to

A grant from the Mountain Plain Consortium funded this study. promote awareness of hyperspectral remote sensing as a viable alternative to terrestrial methods. Agencies will need to train a workforce with the new skills needed to capitalize on these emerging opportunities in remote sensing in general, and hyperspectral analysis in particular. As corporations and nations launch low-cost hyperspectral imaging satellites, and small UAS equipped with hyperspectral image sensors proliferate, new applications in the transportation sector are likely to emerge rapidly.

This study reports on new opportunities to utilize hyperspectral analysis for select applications in transportation that include planning, asset management, network performance evaluation, and risk management. The framework presented generalizes the overall approach and then offers three specific application scenarios to demonstrate new utilities from hyperspectral analysis. The selected scenarios are multimodal. They explain the models used to analyze roadway mobility, railroad energy consumption, and pipeline risk management. The core concept is to identify specific high value applications where both their spatial and spectral characteristics and requirements could benefit from the capabilities of hyperspectral remote sensing.

The global transportation network is expansive, dynamic, multimodal, interdependent, and accommodates a diverse range of human behaviors and needs. Analysts study such complex networks by using models to characterize, understand, and predict their behavior, economics, and risks. The accuracy and utility of those models depend on the quality and frequency of data available to quantify their operational parameters and validate their explanatory variables. Beyond improved modeling capabilities, hyperspectral remote sensing and joint spatial-spectral analysis offers the potential to provide regular and accurate information to enable adaptive transportation infrastructures that would support the future operation, safety, and security of connected and autonomous vehicles.

The organization of the remainder of this paper is as follows: Section 2 introduces a general framework to link transportation systems analysis and hyperspectral analysis. The authors then introduce a range of applications where they foresee high utility for hyperspectral analysis within the general framework. Section 3 presents specific scenario analysis covering three different application areas to demonstrate how the outputs of hyperspectral analysis 
would improve models that practitioners use currently to study a variety of transportation networks. Section 4 discusses the results and concludes the study.

\section{GENERAL METHOD OF APPLICATION}

Practitioners worldwide are seeking cost-effective and simplified tools to help them address numerous problems that arise continuously from the massive, open, dynamic, and multimodal global transportation network. According to data from the Central Intelligence Agency, approximately 40 million miles of roadways, 1.4 million miles of navigable waterways, and more than 40 thousand airports cover the planet's surface. This infrastructure also includes an interacting and interdependent network of railways, pipelines, and shipping ports along with their associated geological and oceanographic structures.

The most serious problems include reducing congestion, energy consumption, polluting emissions, crash risks, and managing the safety and security of hazardous transport. The growing worldwide population and international commerce drives increasing demands for mobility and accessibility across the global network. Hence, solutions must have the capacity to scale internationally, the ability to provide results in real-time, and the propensity for standardization. Hyperspectral remote sensing with fleets of ground-based or UAS offers such an opportunity. Combinations of spatial and spectral data are central to the hyperspectral analysis of scenes from many elements of the global transportation infrastructure. Practitioners with the appropriate training in hyperspectral remote sensing will gain the ability to capitalize on their capabilities and capacity to solve complex transportation problems and enable new applications more cost-effectively. This section introduces a general framework to benefit from hyperspectral remote sensing by linking the analytical methods of transportation systems analysis to outputs of the image analysis.

A first step in the general approach is to identify the parameters of a transportation system model or aspects of the system that are directly observable using machine vision and analysis techniques. Numerous models already exist to study, estimate, and forecast the cost, performance, condition, safety, and security of multimodal and intermodal transportation networks. Existing approaches to estimate the values for observable model parameters require manual visual inspections or expensive terrestrial sensing equipment. Hyperspectral remote sensing using fleets of UAS has the ability and capacity to scale and provide cost-effective data-collection platforms. Small and agile UAS are capable of capturing high spatial-spectral resolution scenes from transportation right-of-ways that include roadways, bridges, waterways, ports, pipelines, and railways.

The proliferation of high-speed wireless standards, low power embedded computing, high-capacity memories, and cloud-computing platforms will continue to provide a steadily improving conduit for metadata data warehousing and analysis. These opportunities will continue to yield both rapid and cost-effective hyperspectral remote sensing and decision-support platforms. Therefore, transportation systems analysis would benefit from a change in paradigm by designing and developing new or improved models that are amenable to machine vision and image analysis methods or by modifying existing models that rely on terrestrial only data formats.

The ability to achieve rapid and wide area coverage with both high spatial and spectral resolution will enable numerous applications in multimodal and intermodal transportation capacity planning, environmental assessment, infrastructure monitoring, safety, and security. Capacity planning encompasses asset management, urban planning, travel demand forecasting, parking demand forecasting, corridor planning, and traffic analysis. Environmental assessment encompasses wetland identification, habitat protection, post disaster management, and historical site assessment. Infrastructure condition and performance monitoring includes roadways, bridges, railways, terminals, pipelines, waterways, seaports, and airports. The area of transportation safety and security encompasses snow and ice detection and removal, toxic spill cleanup, law enforcement, hazard risk management, vulnerability assessment, and emergency response. Numerous parametric or empirical models are available to study and optimize the processes within each of these areas.

\section{SCENARIO STUDIES AND RESULTS}

The next sections will conduct three different scenario analyses in roadway congestion forecasting, railway condition monitoring, and pipeline risk management.

\subsection{Roadway congestion forecasting}

It is possible to use a flow-density model to forecast impending congestion for specific roadway types and geometries by measuring only the traffic density. Researchers still do not have precise closed form models to describe traffic flow and often turn to simulations that use empirical models. Congestion is largely a result of the interaction between vehicles that slow their speed. To understand how remote sensing can provide measurements that computers or planners can use to optimize traffic flow, this section derives the model of stable operating points.

It is convenient to analyze traffic flow in terms of spatial data representing a dynamic supply of vehicle gaps or time-headways along a segment or a bottleneck. The premise of this approach is that trailing distances are a function of speed because humans and computers need to account for reaction time, sight distances, and the deceleration achievable under prevailing conditions. Solving the equations of motion in terms of time-headway $T_{h}$ yields volume $V_{p}$ as the dependent variable where

$$
V_{p}=\frac{T_{h}}{T_{h}^{2}+T_{r} T_{h}-L_{v} / A_{v}}
$$


$T_{r}, A_{v}$, and $L_{v}$ are the reaction time, average vehicle deceleration, and the average length of a vehicle, respectively.

This solution is most relevant to a freeway facility where the selected speed $S_{v}$ is directly proportional to the achievable headway $T_{h}$ such that

$$
S_{v}=-A_{v} T_{h} \text {. }
$$

Practically, speed selection depends on other factors. They include the speed limit and the presence of flow obstructions such as construction, incidents, and slowmoving vehicles in the traffic stream.

The average deceleration achievable also depends on the traffic mix and terrain. For example, the deceleration of a car will be lower if it must match that of a leading truck on a rural two-lane highway that has no passing zones. This analysis removes those constraints to determine the stable operating points in free- and forcedflow traffic.

Intuitively, time-headway extends the space that a vehicle occupies. Hence, as speed increases, vehicles eventually exhaust the available lane capacity. The timeheadways are relatively small at slow speeds and this is why flow increases initially as speed increases. Eventually, the expanding time-headways begin to deplete capacity rapidly and become the dominant factor that actually decreases the vehicle flow rate. Manipulating the equations of motion and flow, and solving for volume as a function of density $D$ yields

$$
V_{p}(D)=\frac{1}{2}\left[\sqrt{A_{v} D\left(A_{v} T_{r}^{2} D+4 L_{v} D-4\right)}+A_{v} T_{r} D\right]
$$

Solving for the peak volume yields

$$
V_{p \max }=\frac{A_{v} T_{r}+2 \sqrt{\left|A_{v}\right| L_{v}}}{A_{v} T_{r}^{2}+4 L_{v}} .
$$

The density $D_{\text {opt }}$ at which the maximum volume occurs is

$$
D_{o p t}=\frac{2 L_{v}-T_{r} \sqrt{\left|A_{v}\right| L_{v}}}{4 L_{v}^{2}+A_{v} L_{v} T_{r}^{2}} .
$$

It is evident an optimum density that would maximize flow exists. Given the average length of a vehicle, the optimum density depends only on the average vehicle deceleration and driver reaction on that facility. Figure 1 plots this non-linear relationship for $L_{v}=5.5$ meters, $A_{v}=-6.5 \mathrm{~m} / \mathrm{s}^{2}$, and $T_{r}=0.66$ seconds.

The same volume is achievable on either side of the peak value. However, the average speed will be higher in free-flow conditions, which is on the left side of the peak. Traffic transitions into forced-flow conditions as the density increases beyond the peak flow condition. The vehicle speed is the slope of a line from the origin to any point on the volume-density curve. For example, the volume is 1000 vehicles/hour for speed $S_{1}$ and $S_{2}$ but the density of vehicles is higher for the lower speed $S_{2}$. This model assures that having an ability to identify and count vehicles in a remotely captured image will enable the accurate quantification of traffic density and hence the flow conditions.

The ability to identify vehicles from their spectral signatures is a mature field [5][6][7]. The Digital Automotive Image System (DAIS) provides spatial

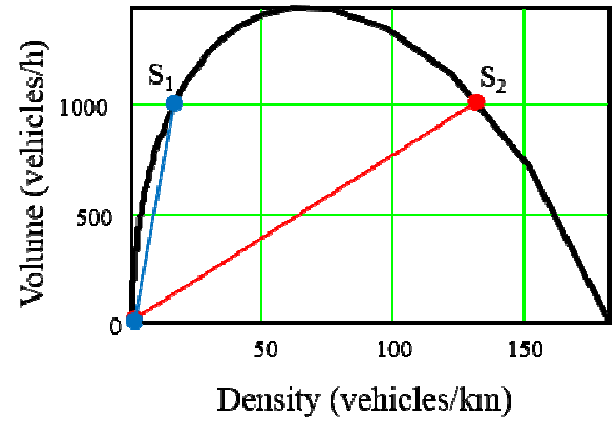

Fig. 1. Theoretical volume-density relationship.

information to identify vehicles manufactured within the past 20 years [8]. New "snapshot" hyperspectral sensors obtain a single spatial-spectral image within a few milliseconds [9]. Hence, they essentially eliminate speedbased distortions and the computational processing typically associated with a pushbroom type systems.

\subsection{Railway condition monitoring}

Energy or fuel consumption is one of the largest factors in the cost of railroad operations, and it is an important consideration in route and locomotive selection. Numerous factors affect railroad energy consumption. They include infrastructure and equipment related considerations. Infrastructure related factors include the line condition, degree of curvature, and the terrain type. Equipment related factors include the energy efficiency of locomotives and rolling resistance. The latter is a function of gross weight, speed, and aerodynamic factors. Remote sensing can help to characterize and catalog line segments of various degrees of curvatures, grades, and other conditions that impede rolling motion to validate existing energy consumption models, and to provide updated inputs to improve them.

A commonly used model for the force needed per ton of railroad car $\mathrm{F}_{\mathrm{RC}}$ is [10]:

$$
F_{R C}=R_{R}+0.8 D_{R}+20 G_{R}
$$

where $R_{R}$ is the rolling resistance on flat terrain, $D_{R}$ is the degree of curvature, and $G_{R}$ is the grade in percentage. All units are in pounds-per-ton (lb/ton). Various models exist that relate the flat-terrain rolling resistance to characteristics of the car. The Canadian National model is most applicable to modern cars that use roller bearings where:

$$
R_{R}=1.5+\frac{18 N_{A C}}{W_{C}}+0.03 V_{R}+\frac{c_{d r} a_{c r}}{10000 \cdot W_{C}} V_{R}^{2}
$$

The first two terms represent the speed-independent resistance, which is a function of track quality and bearing resistance, the number of axles $N_{A C}$, and the total weight of the car $W_{C}$ in tons. The third term is dependent on the vehicle speed $V_{R}(\mathrm{mph})$ where the resistance is a function mostly of wheel-rail interface factors such as flange friction, dynamic flange motion, and wheel resistance. The last term is also speed-dependent; it includes a streamlining coefficient $c_{d r}$ and the frontal car area $a_{c r}$ to 
account for aerodynamic effects such as air pressure, rear drag, and air turbulence.

The high spectral contrast of metal rails and the intervening material such as ballast, soil, and vegetation makes it possible to assess the uniformity of line quality. Uneven wear and rusting will produce variations in their spectral signature to indicate changes in rolling resistance. Irregular spatial geometry on flat terrain such as buckling, kinks, cracks, and railhead abrasions can impede smooth rolling motion. Unmanned Airborne Systems (UAS) based hyperspectral systems with resolution agile snapshot sensors provide a method of simultaneously acquiring images at both the spectral and spectral resolutions needed. Such platforms have the ability to zoom adaptively and obtain higher spatial resolution of target areas within a small field of view. The ability to assess these conditions could provide inputs for improving the rolling resistance model and augment maintenance information to improve railroad safety. The energy required for any route is directly proportional to the sum of forces needed to move the train along various portions of the track having different combinations of rolling resistance, curvature, grade, and speed limit.

\subsection{Pipeline risk management}

The visual identification of toxic spills along pipeline rights-of-way can be nearly impossible because of their changing appearance over time and the limited accessibly of those environments. Fortuitously, the characteristically strong reflectance features of toxic materials such as hydrocarbons and brine make hyperspectral techniques suitable for their detection [11]. Infrared remote sensing has proven effective in the detection of oil spills on open water [12]. The combination of hydrocarbon absorption peaks provides unique signatures for the early detection of spills and the subsequent monitoring of cleanup efforts. The ability to classify hyperspectral scenes in real-time will provide navigational adaptability to guide UAS-based imagers to suspected spill areas for higher resolution image acquisition. However, the computational complexity of existing high performance classifiers limits their ability to provide real-time results [13].

This research introduces a simple spectral classifier (SSC) to demonstrate the separability in feature space of

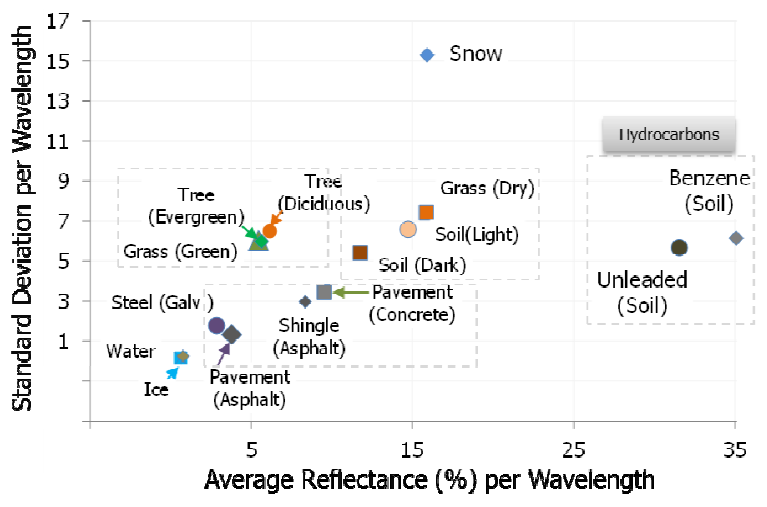

Fig. 2. The SSC feature space for selected materials. hydrocarbon materials from other forms of ground cover. Figure 2 displays the SSC feature space for selected materials sampled from endmembers of the NASA Advanced Spaceborne Thermal Emission Reflection Radiometer (ASTER) Spectral Library [14]. The SSC incorporates the bandwidth normalized albedo and standard deviation of endmember spectral signatures to create a simple two dimensional classification space. The standard deviation $\sigma_{g}$ of the reflectance spectra is

$$
\sigma_{g}=\sqrt{\frac{1}{N} \sum_{n=1}^{N}\left(g_{n}-\mu_{g}\right)^{2}}
$$

where the mean reflectance percentage or albedo $\mu_{g}$ is

$$
\mu_{g}=\frac{1}{N} \sum_{n=1}^{N} g_{n}
$$

The reflectance percentage in spectral band $n$ is $g_{\mathrm{n}}$ and the wavelength-normalized albedo (AVN) is

$$
A V N=\frac{\mu_{g}}{\lambda_{H}-\lambda_{L}}
$$

where $\lambda_{\mathrm{H}}$ and $\lambda_{\mathrm{L}}$ are the highest and lowest wavelength bands, respectively. Similarly, the wavelengthnormalized standard deviation (STN) is

$$
S T N=\frac{\sigma_{g}}{\lambda_{H}-\lambda_{L}}
$$

The physical meaning of the AVN is the reflective nature of a material per unit of wavelength across the spectral region of interest. The STN is a measure of the overall variations in reflectivity of a material per unit of wavelength. The SSC feature space naturally organizes these materials into the six classes shown in the figure. The classes are aquatic, living organics, dry organics, manufactured surfaces, snow, and hydrocarbons. These materials represent a majority of the ground cover in and around typical transportation infrastructures.

At small zenith angles, aquatic materials are highly absorptive throughout the spectral region. This characteristic places the ice and water spectra at an extreme corner of the feature space. Conversely, snow is highly reflective in the visible region and varies in albedo at longer wavelengths. Those features place it in the upper portion of the feature space.

The measure of separability is the Euclidian distance proportion of the maximum distance in the normalized feature space. The Euclidian distance proportion $D_{\eta}$ is

$$
D_{\eta}=\frac{1}{D_{\max }} \sqrt{\left(g_{n x}-h_{n x}\right)^{2}+\left(g_{n y}-h_{n y}\right)^{2}}
$$

where $D_{\max }$ is the maximum Euclidean distance for all features in the normalized space, $\boldsymbol{g}$ and $\boldsymbol{h}$ are vectors of the extracted features for any two materials, $x$ and $y$ are the scaled feature space vector components for the horizontal and vertical axis, respectively.

The average separability for hydrocarbons from the other material classes is $43.4 \%$. Excluding the outlier materials of hydrocarbons and snow to remove the bias provides an average inter-class separability of $22.1 \%$, which is still relatively high. The average intra-class separability, however, is only $3.3 \%$. This result demonstrates that hydrocarbons separate reasonably well 
from the other classes. However, an improved ability to distinguish amongst materials of the same class would likely require a more computational complex algorithm.

This scenario demonstrates the potential for the realtime detection of hazardous spills containing high hydrocarbon and water content by using simple but effective classifiers that offer ample separability between material classes.

\section{DISCUSSION AND CONCLUSION}

The primary benefit derived from using hyperspectral remote sensing to characterize the global transportation infrastructure is the simultaneous spatial and spectral information that it provides about the targets. Typical models of transportation systems analysis utilize information only from the spatial and temporal domains.

Table 1. Typical spatial and spectral requirements.

\begin{tabular}{|c|c|c|c|}
\hline $\begin{array}{l}\text { Application } \\
\text { Resolution } \\
\text { Requirements }\end{array}$ & $\begin{array}{l}\text { Roadway } \\
\text { Congestion } \\
\text { Monitoring }\end{array}$ & $\begin{array}{l}\text { Railway } \\
\text { Condition } \\
\text { Monitoring } \\
\end{array}$ & $\begin{array}{l}\text { Pipeline } \\
\text { Risk } \\
\text { Assessment }\end{array}$ \\
\hline Spatial & $1 \mathrm{~m}$ & $5 \mathrm{~cm}$ & $1 \mathrm{~m}$ \\
\hline Spectral & $\begin{array}{l}15 \mathrm{bands} \\
10 \mathrm{~nm} / \mathrm{band}\end{array}$ & $\begin{array}{l}12 \mathrm{bands} \\
10 \mathrm{~nm} / \mathrm{band}\end{array}$ & $\begin{array}{l}4 \text { bands } \\
50 \mathrm{~nm}\end{array}$ \\
\hline
\end{tabular}

This study introduced a generalized framework for the utilization of both spatial and spectral data to improve and enable many applications in transportation systems. The authors demonstrated the framework within three specific areas where hyperspectral remote sensing provided an appropriate approach to quantify the identified parameters. Table 1 summarizes their expert assessment of conservative spatial and spectral capabilities needed for the three application areas analyzed. Implementation difficulties such as sensor cost, instrumental complexity, payload weight, data archiving, rapid image analysis, and high latencies in the data decision framework are rapidly diminishing. The authors have recently acquired a UAS with procurement procedures in place to accept delivery of a hyperspectral mosaic sensor. The team is currently planning to conduct field-based observations relevant to these three topics. Hyperspectral remote sensing will lead to a paradigm shift in transportation systems modeling.

\section{REFERENCES}

[1] B. J. Rafert, "Before Hyperspectral Ruled the World," in Hyperspectral Imaging and Applications Conference, Glasgow, U.K., 2014.

[2] R. Bridgelall, J. B. Rafert and D. Tolliver, "Hyperspectral Imaging Utility for Transportation Systems," in SPIE Smart Structures/NDE 2015: Sensors and Smart Structures Technologies for Civil, Mechanical, and Aerospace Systems, San Diego, California, 2015.

[3] A. Brecher, V. Noronha and M. Herold, "UAV2003 - A Roadmap for Deploying Unmanned Aerial Vehicles (UAVs) in Transportation," in Volpe Center and NCRST Infrastructure Specialist Workshop (Santa Barbara, California), Cambridge, Massachusetts, 2003.
[4] TRB, "Remote Sensing for Transportation: Products and Results - Foundations for the Future," Transportation Research Board (TRB), Washington, D.C., 2003.

[5] E. M. Suzuki and M. Carrabba, "In Situ Identification and Analysis of Automotive Paint Pigments Using Line Segment Excitation Raman Spectroscopy: I. Inorganic Topcoat Pigments," Journal of Forensic Sciences , vol. 46, no. 5, pp. 1053-1069, 2001.

[6] E. M. McIntee, "Forensic Analysis of Automobile Paints by Atomic and Molecular Spectroscopic Methods and Statistical Data Analyses," University of Central Florida, Orlando, Florida, 2008.

[7] C. S. Palenik, S. Palenik, E. Groves and J. Herb, "Raman Spectroscopy of Automotive and Architectural Paints: In situ Pigment Identification and Evidentiary Significance," Microtrace, LLC, Washington, D.C., 2014.

[8] National Institute of Standards and Technology (NIST), "Forensic Database Trace Evidence Table," NIST, 20134 June. [Online]. Available: http://www.nist.gov/oles/forensics/forensic-database-traceevidence-table.cfm. [Accessed 27 May 2015].

[9] A. Lambrechts, P. Gonzalez, B. Geelen, P. Soussan, K. Tack and M. Jayapala, "A CMOS-Compatible, Integrated Approach to Hyper- and Multispectral Imaging," in Electron Devices Meeting (IEDM), 2014 IEEE International, Piscataway, New Jersey, 2014.

[10] J. H. Armstrong, The Railroad: What it is, What it Does. The Introduction to Railroading, Omaha, Nebraska: Simmons-Boardman Publishing Corporation, 1990, p. 270.

[11] F. Salem and M. Kafatos, "Hyperspectral Image Analysis for Oil Spill Mitigation," in The 22nd Asian Conference on Remote Sensing, Singapore, 2001.

[12] S. Warren, T. Puestow, M. Richard and B. Jefferies, "Oil Spill Detection and Modeling in the Hudson and Davis Straits," LookNorth Center of Excellence for Commercialization and Research, St. John's, Newfoundland, 2014.

[13] M. Borengasser, W. S. Hungate and R. Watkins, Hyperspectral Remote Sensing: Principles and Applications, Boca Raton, Florida: CRC Press, 2010.

[14] A. M. Baldridge, S. Hook, C. Grove and G. Rivera, "The ASTER Spectral Library Version 2.0," Remote Sensing of Environment, vol. 113, pp. 711-715, 2009. 